\title{
DISCRETIZATION OF ASYMPTOTICALLY STABLE STATIONARY SOLUTIONS OF DELAY DIFFERENTIAL EQUATIONS WITH A RANDOM STATIONARY DELAY
}

\author{
TOMÁS CARABALLO, PETER E. KLOEDEN, AND JOSÉ REAL
}

\begin{abstract}
We prove the existence of a stationary random solution to a delay random ordinary differential system which attracts all other solutions in both pullback and forwards senses. The equation contains a one-sided dissipative Lipschitz term without delay, while the random delay appears in a globally Lipschitz one. The delay function only needs to be continuous in time. Moreover, we also prove that the split implicit Euler scheme associated to the random delay differential system generates a discrete time random dynamical system which also possesses a stochastic stationary solution with the same attracting property, and which converges to the stationary solution of the delay random differential equation pathwise as the stepsize goes to zero.
\end{abstract}

\section{INTRODUCTION}

We consider ordinary delay differential equations of the form

$$
\frac{d}{d t} x(t)=F(x(t))+G(x(t-\tau(t, \omega)))
$$

with a random delay $\tau(t, \omega)$ which has continuous sample paths with bounded values $\tau(t, \omega) \in\left[\tau_{*}, \tau^{*}\right]$ where $0 \leq \tau_{*} \leq \tau^{*}$ are finite deterministic numbers and $\omega \in$ $\Omega$ for a given probability space $(\Omega, \mathcal{F}, \mathbb{P})$. In particular, we will be more interested in the case where the delay is a stationary stochastic process, for example $\tau(t, \omega)$ $=1+\cos ^{2} U_{t}(\omega)$ for an Ornstein-Uhlenbeck process $U_{t}$.

The function $F: \mathbb{R}^{d} \rightarrow \mathbb{R}^{d}$ is assumed to be continuously differentiable and satisfy a one-sided dissipative Lipschitz condition

$$
\langle x-y, F(x)-F(y)\rangle \leq-L\|x-y\|_{d}^{2} \text { for all } x, y \in \mathbb{R}^{d},
$$

where $L>0$ and $\|\cdot\|_{d}$ is the Euclidean norm on $\mathbb{R}^{d}$, and function $G: \mathbb{R}^{d} \rightarrow \mathbb{R}^{d}$ is assumed to satisfy the global Lipschitz condition

$$
\|G(x)-G(y)\|_{d} \leq L_{G}\|x-y\|_{d} \text { for all } x, y \in \mathbb{R}^{d} .
$$

Caraballo et al. [4] have considered the existence of nonautonomous pullback attractors for deterministic time-variable delays. Their results apply pathwise here

Key words and phrases. random delay, pullback attractor, stationary solution, split implicit Euler scheme

(2000) Mathematics Subject Classification: 34D45, 34K20, 34K25, 47H20, 58F39, $73 \mathrm{~K} 70$.

Partly supported by Ministerio de Educación y Ciencia (Spain) and FEDER (European Community), project MTM2005-01412. Peter Kloeden has been also supported by the same Ministerio under the grant SAB2004-0146, within the Programa de Movilidad del Profesorado universitario español y extranjero. 
too. Our work here differs in that we use the cocycle rather than process formulation of nonautonomous dynamical systems, which allows us to use the theory of random dynamical systems. In addition, we do not assume that the delay term $G$ is bounded and we use Razumikhin-Lyapunov theory to establish dissipativity of the system which allows to weaken the differentiability assumption on the delay function in [4] to be only continuous.

We will also investigate the effect of time discretization with a constant time step $\Delta>0$ for which $N_{\Delta}:=\tau^{*} / \Delta$ is an integer. Let $t_{n}=n \Delta$ for $n \geq-N_{\Delta}$ and define $N_{n}(\Delta, \omega):=\left\lfloor\tau\left(\theta_{t_{n}} \omega\right) / \Delta\right\rfloor$ (integer part) for $n \geq 0$. We apply to the random DDE (1) the split implicit Euler scheme (SIES)

$$
x_{n+1}=x_{n}+F\left(x_{n+1}\right) \Delta+G\left(x_{n-N_{n}(\Delta, \omega)}\right) \Delta, \quad n=0,1,2, \cdots,
$$

which is essentially a difference equation in $\mathbb{R}^{d}$ of random order or delay requiring initial data

$$
x_{-N_{\Delta}}, x_{-N_{\Delta}+1}, \cdots, x_{-1}, x_{0} .
$$

We will take $x_{-j}:=\phi_{0}(-j \Delta)$ for $j=0,1, \cdots, N_{\Delta}$, where $\phi_{0} \in C\left(\left[-\tau^{*}, 0\right], \mathbb{R}^{d}\right)$ is the initial value under consideration of the random DDE (1).

Our main result is the following.

Theorem 1.1. In addition to the previous assumptions, suppose $L_{G}<L$ and that the delay function is given by $\tau(t, \omega)=\tau\left(\theta_{t} \omega\right)$, where $\theta$ is a metric dynamical system on $\Omega$ representing the noise (see Section 2 below) and $t \mapsto \tau\left(\theta_{t} \omega\right)$ is a continuous function from $\mathbb{R}$ into $\left[\tau_{*}, \tau^{*}\right]$ for each $\omega \in \Omega$. Then, the $D D E$ (1) and the SIES (4) generate, respectively, continuous and discrete time random dynamical systems, each of which has a stochastic stationary solution which pathwise attracts in both forward and pullback senses all other trajectories of their respective equations. Moreover, the numerical stochastic stationary solution converges to that of the DDE pathwise as the stepsize converges to zero.

In Section 2 we recall some background on random dynamical systems. The existence of a stationary solution for the delay system (1) which attracts all other solutions in both the pullback and forwards senses is proved in Section 3. A Razumikhin-Lyapunov argument is exploited in the proofs so that no differentiability of the delay function is needed. Finally, we prove in Section 4 that the split implicit Euler scheme (4) replicates the behaviour of the random DDE (1). The convergence of the numerical attractor to the stationary solution to the DDE (1) is finally established in Section 5 .

\section{RANDOM DYNAMICAL SYSTEMS}

We need some background notation and results. Let $(\Omega, \mathcal{F}, \mathbb{P})$ be a probability space and let $\left(\mathcal{X}, d_{\mathcal{X}}\right)$ be a metric space and let $\mathbb{T}^{+}=\mathbb{R}^{+}$for a continuous time system and $\mathbb{T}^{+}=\mathbb{Z}^{+}$for a discrete time system.

Arnold [1] defined a random dynamical system (RDS) $(\theta, \Phi)$ on $\Omega \times \mathcal{X}$ in terms of a metric dynamical system $\theta$ on $\Omega$, which represents the noise driving the system, and a cocycle mapping $\Phi: \mathbb{T}^{+} \times \Omega \times \mathcal{X} \rightarrow \mathcal{X}$, which represents the dynamics in the 
state space $\mathcal{X}$ and satisfies the properties

1). $\Phi\left(0, \omega, \phi_{0}\right)=\phi_{0}$ for all $\phi_{0} \in \mathcal{X}$ and $\omega \in \Omega$;

2). $\Phi\left(s+t, \omega, \phi_{0}\right)=\Phi\left(s, \theta_{t} \omega, \Phi\left(t, \omega, \phi_{0}\right)\right)$ for all $s, t \geq 0 ; \phi_{0} \in \mathcal{X}$ and $\omega \in \Omega$;

3). $\quad\left(t, \phi_{0}\right) \mapsto \Phi\left(t, \omega, \phi_{0}\right)$ is continuous for each $\omega \in \Omega$;

4). $\quad \omega \mapsto \Phi\left(t, \omega, \phi_{0}\right)$ is $\mathcal{F}$-measurable for all $\left(t, \phi_{0}\right) \in \mathbb{T}^{+} \times \mathcal{X}$.

For example, for an Ito stochastic differential equation on $\mathbb{R}^{d}$ driven by a twosided scalar Wiener process $W_{t}$, i.e. defined for all $t \in \mathbb{R}, \theta$ is defined by $\theta_{t} \omega(\cdot)=$ $\omega(t+\cdot)-\omega(\cdot)$ on a canonical sample space $\Omega=C_{0}(\mathbb{R}, \mathbb{R})$ (the space of continuous functions from $\mathbb{R}$ into itself and vanishing at zero) and $\Phi$ on the the space $\mathcal{X}=\mathbb{R}^{d}$ is defined by $\Phi\left(t, \omega, x_{0}\right)=X\left(t ; 0, \omega, x_{0}\right)$, the solution of the SDE starting at $x_{0}$ at time 0 . See [1] for more details. In what follows, we consider as our probability space the canonical one described above.

Although a more general expression for the random delay could be possible, in this paper we only consider a time dependent random delay of the form $\tau(t, \omega):=$ $\tau\left(\theta_{t} \omega\right)$ where $\tau: \Omega \rightarrow\left[\tau_{*}, \tau^{*}\right]$, for some finite $\tau^{*} \geq \tau_{*} \geq 0$, is a given $\mathcal{F}$-measurable function. Then, the solutions of the random delay DE (1) generate a continuous time $\operatorname{RDS} \Phi(\cdot, \cdot, \cdot)$ on the Banach state space $\mathcal{X}=C\left(\left[-\tau^{*}, 0\right], \mathbb{R}^{d}\right)$ with the norm $\|\phi\|_{\mathcal{X}}=\max _{-\tau^{*} \leq s \leq 0}\|\phi(s)\|_{d}$. To fix our notation, we denote by $x\left(\cdot ; t_{0}, \omega, \phi\right)$ the solution of the following problem

$$
\left\{\begin{array}{l}
\frac{d}{d t} x(t)=F(x(t))+G\left(x\left(t-\tau\left(\theta_{t} \omega\right)\right)\right), \quad t \geq 0, \\
x\left(t_{0}+s\right)=\phi(s), \quad s \in\left[-\tau^{*}, 0\right]
\end{array}\right.
$$

where $\phi \in \mathcal{X}$. For $t \geq 0$, we denote by $x_{t}\left(\cdot ; t_{0}, \omega, \phi\right)$ the element in $C\left(\left[-\tau^{*}, 0\right], \mathbb{R}^{d}\right)$ defined by

$$
x_{t}\left(s ; t_{0}, \omega, \phi\right)=x\left(t+s ; t_{0}, \omega, \phi\right), \quad s \in\left[-\tau^{*}, 0\right] .
$$

Then, $\Phi$ is defined as follows:

$$
\begin{aligned}
\Phi & : \mathbb{R}_{+} \times \Omega \times \mathcal{X} \longrightarrow \mathcal{X} \\
(t, \omega, \phi) & \mapsto \Phi(t, \omega, \phi):=x_{t}(\cdot ; 0, \omega, \phi) .
\end{aligned}
$$

The cocycle mapping properties $1-3$ above follow pathwise as in the nonrandom variable case considered in [4], while the measurability property 4 follows from the fact that the projection $x(t)=\Phi\left(t, \omega, \phi_{0}\right)(0)$ in $\mathbb{R}^{d}$ of the solution $\Phi\left(t, \omega, \phi_{0}\right)$ in $\mathcal{X}$ satisfies the integral equation

$$
x(t)=x(0)+\int_{0}^{t} F(x(s)) d s+\int_{0}^{t} G\left(x\left(s-\tau\left(\theta_{s} \omega\right)\right)\right) d s,
$$

the right hand side of which is measurable in $\omega$ in view of the measurability of $\tau$ and $\theta_{t}$, the continuity of the solution and the mapping $G$. Similarly, the split implicit Euler scheme (4) with constant stepsize $\Delta$ applied to the random delay DE (1) generates a discrete time RDS on the state space $\mathbb{R}^{\left(1+N_{\Delta}\right) d}$ with dimension depending on the stepsize $\Delta$. 
A family $\widehat{A}=\{A(\omega), \omega \in \Omega\}$ of nonempty measurable compact subsets of $\mathcal{X}$ is called $\Phi$-invariant if $\Phi(t, \omega, A(\omega))=A\left(\theta_{t} \omega\right)$ for all $t \geq 0$ and is called a random attractor if in addition it is pathwise pullback attracting in the sense that

$$
H_{\mathcal{X}}^{*}\left(\Phi\left(t, \theta_{-t} \omega, D\left(\theta_{-t} \omega\right)\right), A(\omega)\right) \rightarrow 0 \quad \text { as } \quad t \rightarrow+\infty
$$

for all suitable (i.e. in a given attracting universe) families of $\widehat{D}=\{D(\omega), \omega \in \Omega\}$ of nonempty measurable bounded subsets of $\mathcal{X}$. Here $H_{\mathcal{X}}^{*}$ is the Hausdorff semidistance between the closed and bounded subsets of $\mathcal{X}$. The following result [1] ensures the existence of a random attractor for RDS on a metric space.

Theorem 2.1. Let $(\theta, \Phi)$ be a continuous or discrete time $R D S$ on $\Omega \times \mathcal{X}$ such that $\Phi(t, \omega, \cdot): \mathcal{X} \rightarrow \mathcal{X}$ is a compact operator for each fixed $t>0$ and $\omega \in \Omega$. If there exists a family $\widehat{B}=\{B(\omega), \omega \in \Omega\}$ of nonempty measurable closed and bounded subsets of $\mathcal{X}$ and a $T_{\widehat{D}, \omega} \geq 0$ such that

$$
\Phi\left(t, \theta_{-t} \omega, D\left(\theta_{-t} \omega\right)\right) \subset B(\omega), \quad \forall t \geq T_{\widehat{D}, \omega}
$$

for all families $\widehat{D}=\{D(\omega), \omega \in \Omega\}$ in the given attracting universe, then the RDS $(\theta, \Phi)$ has a random attractor $\widehat{A}=\{A(\omega), \omega \in \Omega\}$ with the component subsets defined for each $\omega \in \Omega$ by

$$
A(\omega)=\bigcap_{s>0}{\overline{\bigcup_{t \geq s} \Phi\left(t, \theta_{-t} \omega, B\left(\theta_{-t} \omega\right)\right)}}_{d \mathcal{X}} .
$$

The family $B(\omega)$ is called a pullback absorbing family for the RDS.

Note that if the random attractor consists of singleton sets, i.e $A(\omega)=\left\{X^{*}(\omega)\right\}$ for some random variable $X^{*}$ with $X^{*}(\omega) \in \mathcal{X}$, then $X^{*}(t, \omega):=X^{*}\left(\theta_{t} \omega\right)$ is a stationary stochastic process on $\mathcal{X}$.

\section{Stationary stochastic solutions of the DDE}

We first show that the delay system (1) is pathwise "extremely stable" in the terminology of Yoshizawa. Thus all solutions converge together - then we will use the theory of random dynamical systems to show that they converge to a stochastic stationary solution. To prove this fact we will use a Razumikhin-Lyapunov type argument which is an improvement of some results in [11] which is also mentioned in [12]. We also need the following lemma.

Lemma 3.1. Let $x(\cdot)$ and $y(\cdot)$ be two solutions to the random DDE (1). Then, denoting by $R(t):=\|x(t)-y(t)\|_{d}$, it follows that

$$
\frac{d}{d t} R^{2}(t) \leq-2 L R^{2}(t)+2 L_{G} R(t) R(t-\tau(t)), \quad \forall t \geq 0 .
$$


Proof. Let us fix $\omega \in \Omega$ and write $\tau(t)$ instead of $\tau\left(\theta_{t} \omega\right)$. Observe that, thanks to our assumptions, if $x(\cdot)$ and $y(\cdot)$ are two solutions to the random DDE (1), we have

$$
\begin{aligned}
\frac{d}{d t}\|x(t)-y(t)\|_{d}^{2}=2\left\langle x(t)-y(t), \frac{d}{d t} x(t)-\frac{d}{d t} y(t)\right\rangle \\
=2\langle x(t)-y(t), F(x(t))-F(y(t))\rangle \\
\quad+\langle x(t)-y(t), G(x(t-\tau(t)))-G(y(t-\tau(t)))\rangle \\
\leq-2 L\|x(t)-y(t)\|_{d}^{2} \\
\quad+2 L_{G}\|x(t)-y(t)\|_{d} \| x(t-\tau(t))-y\left(t-\tau(t) \|_{d} .\right.
\end{aligned}
$$

We now have the following result.

Theorem 3.2. If $L_{G}<L$, then $\|x(t)-y(t)\|_{d} \rightarrow 0$ pathwise as $t \rightarrow \infty$ for any two solutions $x(\cdot)$ and $y(\cdot)$ of the random DDE (1).

Proof. Let us fix $\omega \in \Omega$ and write $\tau(t)$ instead of $\tau\left(\theta_{t} \omega\right)$. Now, we consider a fixed solution $y(\cdot)$ to Eq. (1) defined globally in time, and take another arbitrary solution $x(\cdot)$ satisfying the same condition. First, we need to write a differential system which has zero and $x(\cdot)-y(\cdot)$ as solutions. Then, we will prove that zero is a global attractor.

Indeed, let us notice that

$$
\begin{aligned}
\frac{d}{d t}(x(t)-y(t)) & =F(x(t))-F(y(t))+G(x(t-\tau(t)))-G(y(t-\tau(t))), \quad t \geq 0 \\
& =F(y(t)+x(t)-y(t))-F(y(t)) \\
& +G(y(t-\tau(t))+x(t-\tau(t))-y(t-\tau(t)))-G(y(t-\tau(t))) \\
& =\hat{F}(t, x(t)-y(t))+\hat{G}(t, x(t-\tau(t))-y(t-\tau(t))),
\end{aligned}
$$

where the functions $\hat{F}$ and $\hat{G}$ are defined as

$$
\begin{aligned}
& \hat{F}(t, v)=F(y(t)+v)-F(y(t)), \quad t \geq 0, v \in \mathbb{R}^{d}, \\
& \hat{G}(t, v)=G(y(t-\tau(t))+v)-G(y(t-\tau(t))), \quad t \geq 0, v \in \mathbb{R}^{d} .
\end{aligned}
$$

Denoting $u(t)=x(t)-y(t)$, it follows that

$$
\begin{aligned}
\frac{d}{d t} u(t) & =\hat{F}(t, u(t))+\hat{G}(t, u(t-\tau(t))) \\
& =f\left(t, u_{t}\right), \quad t \geq 0,
\end{aligned}
$$

where

$$
f(t, \phi)=\hat{F}(t, \phi(0))+\hat{G}(t, \phi(-\tau(t))), \quad \text { for } \phi \in \mathcal{X}, t \geq 0 .
$$

Now we are going to apply Theorem 4.2 in Hale \& Lunel [12] taking into account that the assumption in this theorem which imposes on $f$ in (8) to take $\mathbb{R}_{+} \times($bounded sets of $\mathcal{X})$ into bounded sets in $\mathbb{R}^{d}$ has been weakened by Burton in $[2]$ to satisfy only that $f$ takes bounded and closed sets from $\mathbb{R}_{+} \times \mathcal{X}$ into bounded sets of $\mathbb{R}^{d}$, which in our case is obviously fulfilled thanks to the continuity of $F$ and 
the Lipschitz continuity of $G$. Then, denoting $R(t)=\|x(t)-y(t)\|_{d}=\|u(t)\|_{d}$, we have from Lemma 3.1

$$
\frac{d}{d t} R^{2}(t) \leq-2 L R^{2}(t)+2 L_{G} R(t) R(t-\tau(t)), \quad t \geq 0 .
$$

Taking now $V(v)=\|v\|_{d}^{2}$ for $v \in \mathbb{R}^{d}$, it follows that $V(u(t))=R^{2}(t)$ and

$$
\begin{aligned}
\frac{d}{d t} V(u(t)) & =\frac{d}{d t} R^{2}(t) \\
& \leq-2 L R^{2}(t)+2 L_{G} R(t) R(t-\tau(t)) \\
& \leq-2\left(L-L_{G}\right) R^{2}(t),
\end{aligned}
$$

provided $V(u(t-\tau(t)))=R^{2}(t-\tau(t))<R^{2}(t)=V(u(t))$.

On the other hand, if we consider again the Lyapunov-Razumikhin function $V(v)=$ $\|v\|_{d}^{2}$ for $v \in \mathbb{R}^{d}$, and the real function $p(s)=q^{2} s, s \in \mathbb{R}_{+}$for some $q>1$ such that $L-q L_{G}>0$ (which is possible since $L-L_{G}>0$ ), we then have

$$
\begin{aligned}
\frac{d}{d t} V(u(t)) & =\frac{d}{d t} R^{2}(t) \\
& \leq-2 L R^{2}(t)+2 L_{G} R(t) R(t-\tau(t)) \\
& \leq-2\left(L-q L_{G}\right) R^{2}(t),
\end{aligned}
$$

provided $p(V(u(t)))=q^{2} R^{2}(t)>V(u(t-\tau(t)))=R^{2}(t-\tau(t))$. Consequently, by Theorem 4.2 in [12] it follows that $u=0$ is a global attractor for the problem (8), and the proof is complete.

We adapt the definition in Hale ([10], page 104) of uniformly ultimately bounded.

Definition 3.3. The random DDE (1) is said to be pathwise uniformly ultimately bounded if there is a $\beta>0$ such that for each $\omega$ and $\alpha>0$ there is a $T_{0}(\omega, \alpha)>0$ such that the solution of (1) satisfies

$$
\left\|x\left(t ; t_{0}, \omega, \phi\right)\right\|_{d} \leq \beta \quad \text { for } t \geq t_{0}+T_{0}(\omega, \alpha)
$$

for all $t_{0} \in \mathbb{R}$ and $\phi \in \mathcal{X}$ with $\|\phi\|_{\mathcal{X}} \leq \alpha$.

The following theorem will also be proved using Razumikhin-Lyapunov arguments.

Theorem 3.4. If $L_{G}<L$, then the random DDE (1) is pathwise uniformly ultimately bounded.

Proof. ¿From assumptions (2) and (3) with $y=0$ we have

$$
\langle x, F(x)\rangle \leq-L\|x\|_{d}^{2}+\|x\|_{d}\|F(0)\|_{d} \text { for all } x \in \mathbb{R}^{d},
$$

and

$$
\| G(x))\left\|_{d} \leq L_{G}\right\| x\left\|_{d}+\right\| G(0) \|_{d} \quad \text { for all } x \in \mathbb{R}^{d} .
$$


Writing $\tau(t)$ again instead of $\tau\left(\theta_{t} \omega\right)$ and using the inequalities (9)-(10) we have

$$
\begin{aligned}
\frac{d}{d t}\|x(t)\|_{d}^{2} & =2\left\langle x(t), \frac{d}{d t} x(t)\right\rangle \\
& =2\langle x(t), F(x(t))\rangle+2\langle x(t), G(x(t-\tau(t)))\rangle \\
& \leq-2 L\|x(t)\|_{d}^{2}+2\|x(t)\|_{d}\|F(0)\|_{d}+2\|x(t)\|_{d}\left(L_{G}\|x(t-\tau(t))\|_{d}+\|G(0)\|_{d}\right) \\
& =-2 L\|x(t)\|_{d}^{2}+2 L_{G}\|x(t)\|_{d}\|x(t-\tau(t))\|_{d}+2\|x(t)\|_{d}\left(\|F(0)\|_{d}+\|G(0)\|_{d}\right)
\end{aligned}
$$

and, for a small enough $\gamma>0$ such that $L-L_{G}-\gamma>0$,

$$
\frac{d}{d t} R^{2}(t) \leq-2(L-\gamma) R^{2}(t)+2 L_{G} R(t) R(t-\tau(t))+C_{0}
$$

where $R(t):=\|x(t)\|_{d}$ and $C_{0}:=\frac{1}{2 \gamma}\left(\|F(0)\|_{d}+\|G(0)\|_{d}\right)^{2}$.

Now we apply Theorem 4.3 in Hale [12] for each $\omega$. To this end, we take $q>1$ such that $L-L_{G} q-\gamma>0$, define $p(s)=q^{2} s$ for $s>0$, and $w(s)=2\left(L-L_{G} q-\gamma\right) s$ for $s>0$. Now, we observe that

$$
\begin{aligned}
\frac{d}{d t} R^{2}(t) & \leq-2(L-\gamma) R^{2}(t)+2 L_{G} R(t) R(t-\tau(t))+C_{0} \\
& \leq-2\left(L-\gamma-L_{G} q\right) R^{2}(t)+C_{0}
\end{aligned}
$$

provided $R^{2}(t-\tau(t))<p\left(R^{2}(t)\right)$. In addition, there exists $H \geq 0$ such that

$$
\begin{aligned}
\frac{d}{d t} R^{2}(t) & \leq-2\left(L-\gamma-L_{G} q\right) R^{2}(t)+C_{0} \\
& \leq-2\left(L-\gamma-L_{G} q\right) R(t),
\end{aligned}
$$

provided that $R(t) \geq H$. This completes the proof.

Since we have uniform ultimate boundedness and, as one can prove by straightforward computations that

$$
x\left(t ; t_{0}, \theta_{t_{1}} \omega, \phi\right)=x\left(t+t_{1} ; t_{0}+t_{1}, \omega, \phi\right),
$$

for $t, t_{0}, t_{1} \in \mathbb{R}, \omega \in \Omega, \phi \in \mathcal{X}$, then we have that $T_{0}\left(\theta_{t} \omega, \alpha\right) \equiv T_{0}(\omega, \alpha)$ for all $t \in$ $\mathbb{R}$. Indeed, observe that the uniform ultimate boundedness implies that

$$
\left\|x\left(t ; t_{0}, \omega, \phi\right)\right\|_{d} \leq \beta \text { for } t \geq t_{0}+T_{0}(\omega, \alpha) .
$$

Applying this definition for $\theta_{t_{1}} \omega$ instead of $\omega$, we have

$$
\left\|x\left(t ; t_{0}, \theta_{t_{1}} \omega, \phi\right)\right\|_{d} \leq \beta \quad \text { for } t \geq t_{0}+T_{0}\left(\theta_{t_{1}} \omega, \alpha\right),
$$

but, thanks to (12), we should have

$$
\left\|x\left(t ; t_{0}, \theta_{t_{1}} \omega, \phi\right)\right\|_{d}=\left\|x\left(t+t_{1} ; t_{0}+t_{1}, \omega, \phi\right)\right\|_{d} \leq \beta \quad \text { for } t \geq t_{0}+T_{0}\left(\theta_{t_{1}} \omega, \alpha\right)
$$

and

$$
\left\|x\left(t+t_{1} ; t_{0}+t_{1}, \omega, \phi\right)\right\|_{d} \leq \beta \quad \text { for } t+t_{1} \geq t_{0}+t_{1}+T_{0}(\omega, \alpha),
$$

so $T_{0}\left(\theta_{t_{1}} \omega, \alpha\right)=T_{0}(\omega, \alpha)$ for any $t_{1} \in \mathbb{R}$.

Hence we have both pullback and forward ultimate boundedness, i.e. absorbing sets, for each fixed $\omega$ and we can take $B(\omega)$ to be the ball of radius $\beta$ about the origin in the space $\mathcal{X}$. 
The solution mapping $\Phi(t, \omega, \cdot)$ of our pathwise random DDE (1) is compact from the space $\mathcal{X}$ into itself for each $t>0$ and $\omega \in \Omega$. This follows from the fact that the solutions are Lipschitz in time even if the initial value function $\phi_{0} \in C\left(\left[-\tau^{*}, 0\right], \mathbb{R}\right)$ is only continuous, i.e.

$$
\|x(t+\rho)-x(t)\|_{d} \leq \int_{t}^{t+\rho}\|F(x(s))\|_{d} d s+\int_{t}^{t+\rho}\|G(x(s-\tau(s)))\|_{d} d s \leq K \rho
$$

for $t, \rho \geq 0$, where $K:=\max _{x \in C}\|F(x)\|_{d}+\max _{x \in C}\|G(x)\|_{d}$ and $C$ is a compact subset of $\mathbb{R}^{d}$ which contains the DDE solution over the time interval $[0, T]$.

Hence the pathwise random DDE (1) has a random attractor which pathwise attracts all bounded subsets in both the pullback and forward senses.

By Theorem 3.2 all solutions converge together as time increases, which means that the random attractor consists of singleton sets, i.e. is a stochastic stationary solution $X^{*}(\cdot) \in \mathcal{X}$. It is pathwise asymptotically attracting in both the pullback and forward senses.

\section{Stationary stochastic solutions of the SiES}

Our aim here is to show that the split implicit Euler scheme (SIES) (4) replicates the behaviour of the random DDE (1) projected into $\mathbb{R}^{d}$, in particular that it has a unique entire solution $\left\{x_{n}^{*}(\omega), n \in \mathbb{Z}\right\}$, which attracts all other solutions pathwise in both forwards and pullback senses, and is stationary in the sense that the $N_{\Delta} d$-dimensional random vectors $X_{n, \Delta}^{*}$ with realisations $X_{n, \Delta}^{*}(\omega)$ $=\left(x_{n-N_{\Delta}}^{*}(\omega), x_{n-N_{\Delta}+1}^{*}(\omega), \cdots, x_{n}^{*}(\omega)\right)^{\top}$ are stationarily distributed in $\mathbb{R}^{\left(1+N_{\Delta}\right) d}$. Moreover, we will show that the $X_{0, \Delta}^{*}$ converge in an appropriate sense to $X^{*}(\omega)$ for each $\omega \in \Omega$ as $\Delta \rightarrow 0$. For this we will show that the SIES generates a discretetime random dynamical system on $\mathbb{R}^{\left(1+N_{\Delta}\right) d}$.

We need discrete time analogues of Theorems 3.2 and 3.4. As in their proofs we have the following inequalities. For notational brevity we write $N_{n}(\omega)$ for $N_{n}(\Delta, \omega)$ when $\Delta$ is fixed.

Lemma 4.1. Any two solutions $x_{n}$ and $y_{n}$ of the SIES (4) satisfy

$$
\begin{aligned}
\left\|x_{n+1}-y_{n+1}\right\|_{d} & \leq \frac{1}{1+L \Delta}\left\|x_{n}-y_{n}\right\|_{d}+\frac{L_{G} \Delta}{1+L \Delta}\left\|x_{n-N_{n}(\omega)}-y_{n-N_{n}(\omega)}\right\|_{d} \\
\left\|x_{n+1}\right\|_{d} \leq & \frac{1}{1+L \Delta}\left\|x_{n}\right\|_{d}+\frac{L_{G} \Delta}{1+L \Delta}\left\|x_{n-N_{n}(\omega)}\right\|_{d} \\
& +\frac{\Delta}{1+L \Delta}\left(\|F(0)\|_{d}+\|G(0)\|_{d}\right)
\end{aligned}
$$

for $n=0,1,2, \cdots$. 
Proof. The proof of the first inequality follows from the fact that

$$
\begin{gathered}
\left\|x_{n+1}-y_{n+1}\right\|_{d}^{2}=\left\langle x_{n+1}-y_{n+1}, x_{n}-y_{n}\right\rangle+\left\langle x_{n+1}-y_{n+1}, F\left(x_{n+1}\right)-F\left(y_{n+1}\right)\right\rangle \Delta \\
+\left\langle x_{n+1}-y_{n+1}, G\left(x_{n-N_{n}(\omega)}\right)-G\left(y_{n-N_{n}(\omega)}\right)\right\rangle \Delta \\
\leq\left\|x_{n+1}-y_{n+1}\right\|_{d}\left\|x_{n}-y_{n}\right\|_{d}-L \Delta\left\|x_{n+1}-y_{n+1}\right\|_{d}^{2} \\
\quad+L_{G} \Delta\left\|x_{n+1}-y_{n+1}\right\|_{d}\left\|x_{n-N_{n}(\omega)}-y_{n-N_{n}(\omega)}\right\|_{d},
\end{gathered}
$$

while the proof of the second inequality follows from the fact that

$$
\begin{gathered}
\left\|x_{n+1}\right\|_{d}^{2}=\left\langle x_{n+1}, x_{n}\right\rangle+\left\langle x_{n+1}-0, F\left(x_{n+1}\right)-F(0)\right\rangle \Delta+\left\langle x_{n+1}, F(0)\right\rangle \Delta \\
+\left\langle x_{n+1}-0, G\left(x_{n-N_{n}(\omega)}\right)-G(0)\right\rangle \Delta+\left\langle x_{n+1}, G(0)\right\rangle \Delta \\
\leq\left\|x_{n+1}\right\|_{d}\left\|x_{n}\right\|_{d}-L \Delta\left\|x_{n+1}\right\|_{d}^{2}+\left\|x_{n+1}\right\|_{d}\|F(0)\|_{d} \Delta \\
+L_{G} \Delta\left\|x_{n+1}\right\|_{d}\left\|x_{n-N_{n}(\omega)}\right\|_{d}+\left\|x_{n+1}\right\|_{d}\|G(0)\|_{d} \Delta .
\end{gathered}
$$

Theorem 4.2. Suppose that $L_{G}<L$. Then $\left\|x_{n}-y_{n}\right\|_{d} \rightarrow 0$ as $n \rightarrow \infty$ for two solutions $x_{n}$ and $y_{n}$ of the SIES (4).

Proof. Define $R_{n}:=\left\|x_{n}-y_{n}\right\|_{d} \geq 0$ for $n \geq-N_{\Delta}$. The first inequality of Lemma 4.1 then reads

$$
R_{n+1} \leq \gamma(\Delta) R_{n}+\gamma(\Delta) L_{G} \Delta R_{n-N_{n}(\omega)}, \quad n \geq 1,
$$

where

$$
\gamma(\Delta):=\frac{1}{1+L \Delta}
$$

Suppose that the initial values $R_{-j} \in[0, B]$ for $j=0,1, \ldots, N_{\Delta}$ and define $\eta(\Delta)$ $:=\gamma(\Delta)\left(1+L_{G} \Delta\right)$, so clearly $\gamma(\Delta)<\eta(\Delta)<1$. Then

$$
R_{1} \leq \gamma(\Delta) R_{0}+\gamma(\Delta) L_{G} \Delta R_{-N_{0}(\omega)} \leq \eta(\Delta) B<B
$$

Repeating this argument inductively we obtain $R_{n} \leq \eta(\Delta) B<B$ for all $n \geq 1$.

Consider the norm

$$
\|\mathbf{x}\|_{\infty}:=\max _{j=0,1, \ldots, N_{\Delta}}\left\|x_{j}\right\|_{d}
$$

on $\mathbb{R}^{\left(1+N_{\Delta}\right)}$ and define $\mathbf{R}_{n}:=\left(R_{n-N_{\Delta}}, \cdots, R_{n}\right)^{\top} \in \mathbb{R}^{\left(1+N_{\Delta}\right)}$ for $n \geq 0$. Then $\left\|\mathbf{R}_{0}\right\|_{\infty} \leq B$ by assumption, hence $\left\|\mathbf{R}_{n}\right\|_{\infty} \leq B$ for $n=1, \cdots, N_{\Delta}$. Thereafter we have

$$
\left\|\mathbf{R}_{N_{\Delta}+1}\right\|_{\infty} \leq \gamma(\Delta)\left\|\mathbf{R}_{N_{\Delta}}\right\|_{\infty}+\gamma(\Delta) L_{G} \Delta\left\|\mathbf{R}_{N_{\Delta}}\right\|_{\infty}=\eta(\Delta)\left\|\mathbf{R}_{N_{\Delta}}\right\|_{\infty}
$$

and it follows inductively that

$$
\left\|\mathbf{R}_{N_{\Delta}+j}\right\|_{\infty} \leq \eta(\Delta)^{j}\left\|\mathbf{R}_{N_{\Delta}}\right\|_{\infty} \leq \eta(\Delta)^{j} B
$$

for $j \geq 1$. Thus $\left\|\mathbf{R}_{n}\right\|_{\infty} \rightarrow 0$ as $n \rightarrow \infty$, which completes the proof of the theorem.

Theorem 4.3. Suppose that $L_{G}<L$. Then the SIES (4) is pathwise uniformly ultimately bounded. 
Proof. Define $R_{n}:=\left\|x_{n}\right\|_{d} \geq 0$ for $n \geq-N_{\Delta}$, so $R_{n} \geq 0$ for $n \geq-N_{\Delta}$. The second inequality of Lemma 4.1 then reads

$$
R_{n+1} \leq \gamma(\Delta) R_{n}+\gamma(\Delta) L_{G} \Delta R_{n-N_{n}(\omega)}+\gamma(\Delta) \Delta C_{0}, \quad n \geq 1,
$$

where $C_{0}:=\|F(0)\|_{d}+\|G(0)\|_{d}$.

Suppose that the initial values $R_{-j} \in[0, B]$ for $j=0,1, \ldots, N_{\Delta}$. Then

$$
\begin{aligned}
R_{1} & \leq \gamma(\Delta) R_{0}+\gamma(\Delta) L_{G} \Delta R_{-N_{0}(\omega)}+\gamma(\Delta) \Delta C_{0} \\
& \leq \eta(\Delta) B+\gamma(\Delta) \Delta C_{0} \leq B+\gamma(\Delta) \Delta C_{0} .
\end{aligned}
$$

Then

$$
R_{2} \leq \eta(\Delta)\left(B+\gamma(\Delta) \Delta C_{0}\right)+\gamma(\Delta) \Delta C_{0}<B+2 \gamma(\Delta) \Delta C_{0}
$$

and repeating this argument inductively we obtain $R_{n} \leq B+n \gamma(\Delta) \Delta C_{0} \leq B+$ $\gamma(\Delta) C_{0} \tau^{*}$ for $n=1, \ldots, N_{\Delta}$, since $N_{\Delta} \Delta=\tau^{*}$.

Now define $\mathbf{R}_{n}:=\left(R_{n-N_{\Delta}}, \cdots, R_{n}\right)^{\top} \in \mathbb{R}^{\left(1+N_{\Delta}\right)}$ for $n \geq 0$. Then $\left\|\mathbf{R}_{0}\right\|_{\infty} \leq B$ by assumption and $\left\|\mathbf{R}_{n}\right\|_{\infty} \leq B+\gamma(\Delta) C_{0} \tau^{*}$ for $n=1, \cdots, N_{\Delta}$ from above. Thereafter we have

$$
\left\|\mathbf{R}_{N_{\Delta}+j+1}\right\|_{\infty} \leq \gamma(\Delta)\left\|\mathbf{R}_{N_{\Delta}+j}\right\|_{\infty}+\gamma(\Delta) C_{0} \Delta
$$

and it follows inductively that

$$
\left\|\mathbf{R}_{N_{\Delta}+j}\right\|_{\infty} \leq \eta(\Delta)^{j}\left(B+\gamma(\Delta) C_{0} T\right)+\frac{\gamma(\Delta) C_{0} \Delta}{1-\eta(\Delta)}=\eta(\Delta)^{j}\left(B+N_{\Delta}\right)+\frac{C_{0}}{L-L_{G}}
$$

for $j \geq 1$. Thus

$$
\left\|\mathbf{R}_{N_{\Delta}+j}\right\|_{\infty} \leq 1+\frac{C_{0}}{L-L_{G}}
$$

for all $j \geq J_{B, \Delta}$, where

$$
J_{B, \Delta}:=1+\left\lfloor\frac{\ln \frac{1}{B+\gamma(\Delta) C_{0} \tau^{*}}}{\ln \eta(\Delta)}\right\rfloor \quad \text { (integer part). }
$$

Note that the time $N_{\Delta}+J_{B, \Delta}$ to be absorbed into the ball of radius $1+\frac{C_{0}}{L-L_{G}}$ centered on the origin is independent of $\omega$. This completes the proof of the theorem.

We now observe that the SIES generates a discrete time random dynamical system on the state space $\mathcal{X}:=\mathbb{R}^{\left(1+N_{\Delta}\right) d}$ with cocycle mapping $\Phi_{\Delta}: \mathbb{Z}^{+} \times \Omega \times \mathbb{R}^{\left(1+N_{\Delta}\right) d}$ $\rightarrow \mathbb{R}^{\left(1+N_{\Delta}\right) d}$ defined by

$$
\mathbf{X}_{n}:=\Phi_{\Delta}\left(n, \omega, \mathbf{X}_{0}\right)
$$

where $\mathbf{X}_{n}:=\left(x_{n-N_{\Delta}}, \cdots, x_{n}\right)^{\top}$ for $n \geq 0$. As the driving system we take the discrete time metric system formed by iterating $\theta_{\Delta}$ on $\Omega$. RDS theory (see, e.g. $[6],[8],[9],[15])$ then says that we have a unique singleton set pullback attractor $\left\{\mathbf{X}_{\Delta}^{*}\left(\theta_{n \Delta} \omega\right)\right\}$ formed by a stochastic stationary solution $\mathbf{X}_{\Delta}^{*}(\omega) \in \mathbb{R}^{\left(1+N_{\Delta}\right) d}$, which is obviously given by $\mathbf{X}_{\Delta}^{*}(\omega)=\left(X_{\Delta}^{*}(\omega), \cdots, X_{\Delta}^{*}(\omega)\right)^{\top}$, where $X_{\Delta}^{*}(\omega)$ is the stationary solution of the system SIES in $\mathbb{R}^{d}$. 


\section{Convergence of the Numerical ATtractor}

We will prove here that $X_{\Delta}^{*}(\omega)$ converges pathwise to $X^{*}(\omega)(0)$ in $\mathbb{R}^{d}$ as $\Delta \rightarrow 0$ (with the $\Delta$ dividing $\tau^{*}$ exactly) using a proof by contradiction following [13] using the error bound for the SIES in Theorem 5.1 in the Appendix applied pathwise.

Suppose that there is an $\bar{\omega}$, a sequence $\Delta_{j} \rightarrow 0$ as $j \rightarrow \infty$ and an $\epsilon_{0}>0$ such that

$$
\left\|X_{\Delta_{j}}^{*}(\bar{\omega})-X^{*}(\bar{\omega})(0)\right\|_{d} \geq \epsilon_{0}
$$

The $X_{\Delta}^{*}(\omega)$ belong to a common compact absorbing ball $B$ of radius $1+\frac{C_{0}}{L-L_{G}}$ about the origin, which is independent of both $\omega$ and $\Delta$. Now the family of identical balls $\{B(\omega) \equiv B, \omega \in \Omega\}$ is pullback attracted to the random DDE stationary solution. Thus there is a $T(\bar{\omega})$ such that

$$
\left\|\Phi\left(t, \theta_{-t} \bar{\omega}, \widehat{X}_{\Delta}^{*}\left(\theta_{-t} \bar{\omega}\right)\right)(0)-X^{*}(\bar{\omega})(0)\right\|_{d} \leq \epsilon_{0} / 4
$$

for $t \geq T(\bar{\omega})$ where $\widehat{X}_{\Delta}^{*}\left(\theta_{-t} \bar{\omega}\right) \in C\left(\left[-\tau^{*}, 0\right], \mathbb{R}^{d}\right)$ is the linear interpolation of the numerical stationary solution for stepsize $\Delta$ and is such that $\widehat{X}_{\Delta}^{*}\left(\theta_{-t} \bar{\omega}\right)(0) \in B$.

Let $k_{j}$ be the first integer so that $k_{j} \Delta_{j} \geq T(\bar{\omega})$ for any $j \in \mathbb{N}$. Applying this to $\widehat{X}_{\Delta_{j}}^{*}\left(\theta_{-k_{j} \Delta_{j}} \bar{\omega}\right)$ we then have

$$
\left\|\Phi\left(k_{j} \Delta_{j}, \theta_{-k_{j} \Delta_{j}} \bar{\omega}, \widehat{X}_{\Delta_{j}}^{*}\left(\theta_{-k_{j} \Delta_{j}} \bar{\omega}\right)\right)(0)-X^{*}(\bar{\omega})(0)\right\|_{d} \leq \epsilon_{0} / 4
$$

Now we use the global discretization error of the split implicit Euler scheme from Theorem 5.1 of the Appendix on the interval $[-1-T(\bar{\omega}), 0]$ for the delay path corresponding to $\bar{\omega}$ to obtain

$$
\left\|x_{k_{j}}^{\Delta_{j}}\left(\theta_{-k_{j} \Delta_{j}} \bar{\omega}\right)-\Phi\left(k_{j} \Delta_{j}, \theta_{-k_{j} \Delta_{j}} \bar{\omega}, \widehat{X}_{\Delta_{j}}^{*}\left(\theta_{-k_{j} \Delta_{j}} \bar{\omega}\right)\right)(0)\right\|_{d} \leq \epsilon_{0} / 4
$$

for $j$ large enough, where $x_{k_{j}}^{\Delta_{j}}\left(\theta_{-k_{j} \Delta_{j}} \bar{\omega}\right)$ is the $k_{j}$ th iterate of the split implicit Euler solution with step size $\Delta_{j}$ starting at $X_{\Delta_{j}}^{*}\left(\theta_{-k_{j} \Delta_{j}} \bar{\omega}\right)$. Since this is the numerical stationary solution, we have $x_{k_{j}}^{\Delta_{j}}\left(\theta_{-k_{j} \Delta_{j}} \bar{\omega}\right)=X_{\Delta_{j}}^{*}(\bar{\omega})$, and hence

$$
\left\|X_{\Delta_{j}}^{*}(\bar{\omega})-\Phi\left(k_{j} \Delta_{j}, \theta_{-k_{j} \Delta_{j}} \bar{\omega}, \widehat{X}_{\Delta_{j}}^{*}\left(\theta_{-k_{j} \Delta_{j}} \bar{\omega}\right)\right)(0)\right\|_{d} \leq \epsilon_{0} / 4
$$

We combine this with the preceding estimate to obtain

$$
\left\|X_{\Delta_{j}}^{*}(\bar{\omega})-\bar{X}^{*}(\bar{\omega})(0)\right\|_{d} \leq \epsilon_{0} / 2
$$

which contradicts (14).

\section{APPENDIX: NumERICAL DISCRETIZATION ERROR}

We show here that the SIES (4) has global discretization error on any finite time interval. We will do this for the deterministic version of the delay DE (1). 
Theorem 5.1. If $F$ is continuously differentiable, hence satisfies a local Lipschitz condition, if $G$ satisfies a global Lipschitz condition, and if the delay is a continuous function $\tau: \mathbb{R} \rightarrow\left(0, \tau^{*}\right]$, then split implicit Euler scheme (4) with constant stepsize $\Delta$ which divides $\tau^{*}$ exactly has global discretization error of order at least $1 / 2$ when applied to the DDE (1).

Proof. Let $x(t)$ be the value in $\mathbb{R}^{d}$ of the solution of the DDE (1) for $t \in[0, T]$ and let $x_{n}$ be the corresponding solution of the SIES (4) with constant stepsize $\Delta$ which divides $\tau^{*}$ exactly, i.e. so there is an integer $N_{\Delta}$ such that $N_{\Delta} \Delta=\tau^{*}$. Furthermore let $t_{n}=n \Delta$, let $N_{n}=\left\lfloor\tau\left(t_{n}\right) / \Delta\right\rfloor$ (integer part) and let $x_{\Delta}$ denote the linearly interpolated numerical solution, i.e. with in particular

$$
x_{\Delta}(t)=x_{n}+\left(t-t_{n}\right) F\left(x_{n+1}\right)+\left(t-t_{n}\right) G\left(x_{n-N_{n}}\right), \quad t \in\left[t_{n}, t_{n+1}\right], n \geq 0 .
$$

By continuity of the coefficient functions $F$ and $G$ as well as of the DDE solution, there exists a compact subset $C$ of $\mathbb{R}^{d}$ which contains the DDE and SIES solutions as well as the linearly interpolated SIES solution over the time interval $[0, T]$. The linearly interpolated SIES solution is obviously Lipschitz continuous and there exists a constant $K$ which depends on $T$ but not on $\Delta$ such that

$$
\left\|x_{\Delta}(t)-x_{n}\right\|_{d} \leq K \Delta, \quad\left\|x_{\Delta}(t)-x_{n+1}\right\|_{d} \leq K \Delta,
$$

for $t \in\left[t_{n}, t_{n+1}\right] \subset[0, T]$. We know from (13) that the solutions of the DDE are Lipschitz continuous for $t>0$, so it is no loss of generality to assume that the initial value $\phi_{0} \in C\left(\left[-\tau^{*}, 0\right], \mathbb{R}^{d}\right)$ is already Lipschitz continuous. Hence we can assume that the above inequalities also hold for the linearly interpolated initial data on initial time interval $\left[-\tau^{*}, 0\right]$ as well as that $\left\|x_{\Delta}(t-\tau(t))-\phi_{0}(t-\tau(t))\right\|_{d} \leq K \Delta$ for $t \geq 0$ with $t-\tau(t) \in\left[-\tau^{*}, 0\right]$. Moreover,

$$
\begin{gathered}
x(t)-x_{\Delta}(t)=x\left(t_{n}\right)-x_{\Delta}\left(t_{n}\right)+\int_{t_{n}}^{t}\left[F(x(s))-F\left(x_{n+1}\right)\right] d s \\
+\int_{t_{n}}^{t}\left[G(x(s-\tau(s)))-G\left(x_{n-N_{n}}\right)\right] d s
\end{gathered}
$$

so within the interval $\left(t_{n}, t_{n+1}\right)$ we have

$$
\frac{d}{d t}\left[x(t)-x_{\Delta}(t)\right]=\left[F(x(t))-F\left(x_{n+1}\right)\right]+\left[G(x(t-\tau(t)))-G\left(x_{n-N_{n}}\right)\right] .
$$

Using a superscript on the vectors as the component index, for each $i=1, \ldots, d$, this gives

$$
\left|\frac{d}{d t}\left[x^{i}(t)-x_{\Delta}^{i}(t)\right]\right| \leq\left\|F(x(t))-F\left(x_{n+1}\right)\right\|_{d}+\left\|G(x(t-\tau(t)))-G\left(x_{n-N_{n}}\right)\right\|_{d} .
$$

Now from the (local) Lipschitz condition of $F$ on the set $C$ (with constant $L_{F}$ )we have

$$
\begin{aligned}
\left\|F(x(t))-F\left(x_{n+1}\right)\right\|_{d} & \leq L_{F}\left\|x(s)-x_{n+1}\right\|_{d} \\
& \leq L_{F}\left\|x(t)-x_{\Delta}(t)\right\|_{d}+L_{F}\left\|x_{\Delta}(t)-x_{n+1}\right\|_{d} \\
& \leq L_{F}\left\|x(t)-x_{\Delta}(t)\right\|_{d}+L_{F} K \Delta
\end{aligned}
$$


and from the global Lipschitz condition of $G$

$$
\begin{aligned}
\left\|G(x(t-\tau(t)))-G\left(x_{n-N_{n}}\right)\right\|_{d} \leq & L_{G}\left\|x(t-\tau(t))-x_{n-N_{n}}\right\|_{d} \\
\leq & L_{G}\left\|x(t-\tau(t))-x_{\Delta}(t-\tau(t))\right\|_{d} \\
& \quad+L_{G}\left\|x_{\Delta}(t-\tau(t))-x_{n-N_{n}}\right\|_{d} \\
\leq & L_{G}\left\|x(t-\tau(t))-x_{\Delta}(t-\tau(t))\right\|_{d}+L_{G} K \Delta,
\end{aligned}
$$

where we have used the earlier estimates on the linear interpolations of the numerical solution and initial data.

Defining $R(t):=\left\|x(t)-x_{\Delta}(t)\right\|_{d}$ we have the differential inequality

$$
\left|\frac{d}{d t}\left[x^{i}(t)-x_{\Delta}^{i}(t)\right]\right| \leq\left(L_{F}+L_{G}\right) K \Delta+L_{F} R(t)+L_{G} R(t-\tau(t)),
$$

for each $i=1, \ldots, d$, and all $t \in\left(t_{n}, t_{n+1}\right)$ and $n$ with $n \Delta \leq T$.

Now for $\rho_{i}(t)=\left[x^{i}(t)-x_{\Delta}^{i}(t)\right]$ we have

$$
\frac{d}{d t} \rho_{i}(t)^{2}=2 \rho_{i}(t) \frac{d}{d t} \rho_{i}(t) \leq 2\left|\rho_{i}(t)\right|\left|\frac{d}{d t} \rho_{i}(t)\right|
$$

from which it follows that

$$
\frac{d}{d t} R(t)^{2}=\frac{d}{d t} \sum_{i=1}^{d} \rho_{i}(t)^{2} \leq 2 R(t) \sum_{i=1}^{d}\left|\frac{d}{d t} \rho_{i}(t)\right|
$$

and hence that

$$
\frac{d}{d t} R(t)^{2} \leq 2 d\left(L_{F}+L_{G}\right) K R(t) \Delta+2 d L_{F} R(t)^{2}+2 d L_{G} R(t-\tau(t)) R(t)
$$

Finally, we obtain the differential inequality

$$
\frac{d}{d t} R(t)^{2} \leq d\left[L_{F}+L_{G}\right] K \Delta^{2}+d\left[L_{F}(K+2)+L_{G}(K+1)\right] R(t)^{2}+d L_{G} R(t-\tau(t))^{2}
$$

for $t \in\left(t_{n}, t_{n+1}\right)$ and $n$ such that $n \Delta \leq T$.

Now,define

$$
R_{n}(\Delta)^{2}:=\max _{-\tau^{*} \leq t \leq t_{n}} R(t)^{2}, \quad n=0,1,2 \ldots
$$

¿From the assumption above that the initial value $\phi_{0} \in C\left(\left[-\tau^{*}, 0\right], \mathbb{R}^{d}\right)$ is Lipschitz continuous, we have $R(t-\tau(t)) \leq K \Delta$ for $t \geq 0$ with $t-\tau(t) \in\left[-\tau^{*}, 0\right]$, so $R_{0}(\Delta)^{2}$ $\leq K^{2} \Delta^{2}$. Moreover, the differential inequality (15) for $t \in\left[t_{n}, t_{n+1}\right]$ simplifies to

$$
\frac{d}{d t} R(t)^{2} \leq d\left[L_{F}+L_{G}\right] K \Delta^{2}+d\left[L_{F}(K+2)+L_{G}(K+1)\right] R(T)^{2}+d L_{G} R_{n}(\Delta)^{2}
$$

or

$$
\frac{d}{d t} R(t)^{2} \leq d\left(\left[L_{F}+L_{G}\right] K \Delta^{2}+L_{G} R_{n}(\Delta)^{2}\right)+d\left[L_{F}(K+2)+L_{G}(K+1)\right] R(t)^{2} .
$$


The Gronwall inequality with the initial condition $R\left(t_{n}\right)^{2}$ then yields

$$
\begin{aligned}
& R(t)^{2} \leq R\left(t_{n}\right)^{2} e^{L^{*} \Delta}+\frac{\left[L_{F}+L_{G}\right] K}{L_{F}(K+2)+L_{G}(K+1)} \Delta^{2}\left(e^{L^{*} \Delta}-1\right) \\
& {[2 e x] \quad+\frac{L_{G}}{L_{F}(K+2)+L_{G}(K+1)} R_{n}(\Delta)^{2}\left(e^{L^{*} \Delta}-1\right)} \\
& {[2 e x] \leq R_{n}(\Delta)^{2} e^{L^{*} \Delta}+K^{2} \Delta^{2}\left(e^{L^{*} \Delta}-1\right)+R_{n}(\Delta)^{2}\left(e^{L^{*} \Delta}-1\right)}
\end{aligned}
$$

where we have assumed without loss of generality that $K \geq 1$ and set $L^{*}:=$ $d\left[L_{F}(K+2)+L_{G}(K+1)\right]$, so

$$
\frac{L_{F}+L_{G}}{L_{F}(K+2)+L_{G}(K+1)} \leq 1, \quad \frac{L_{G}}{L_{F}(K+2)+L_{G}(K+1)} \leq 1 .
$$

Taking the maximum over the interval $\left[t_{n}, t_{n+1}\right]$ gives the difference inequality

$$
R_{n+1}(\Delta)^{2} \leq R_{n}(\Delta)^{2}\left(2 e^{L^{*} \Delta}-1\right)+K^{2} \Delta^{2}\left(e^{L^{*} \Delta}-1\right)
$$

since the right hand side is larger than $R_{n}(\Delta)^{2}$ and

$$
R_{n+1}(\Delta)^{2}=\max \left\{\max _{t_{n} \leq t \leq t_{n+1}} R(t)^{2}, R_{n}(\Delta)^{2}\right\} .
$$

The solution of this difference inequality is bounded above by

$$
\begin{aligned}
R_{n}(\Delta)^{2} & \leq R_{0}(\Delta)^{2}\left(2 e^{L^{*} \Delta}-1\right)^{n}+K^{2} \Delta^{2} \frac{\left(2 e^{L^{*} \Delta}-1\right)^{n+1}-1}{\left(2 e^{L^{*} \Delta}-1\right)-1} \\
{[2 e x] } & \leq K^{2} \Delta^{2}\left(e^{3 L^{*} \Delta}\right)^{n}+\frac{K^{2} \Delta}{2 L^{*}}\left(\left(e^{3 L^{*} \Delta}\right)^{n+1}-1\right)
\end{aligned}
$$

since $R_{0}(\Delta)^{2} \leq K^{2} \Delta^{2}$ and also $L^{*} \Delta \leq e^{L^{*} \Delta}-1$ and $2 e^{L^{*} \Delta}-1 \leq e^{3 L^{*} \Delta}$ for all $\Delta$ $>0$. Simplifying then gives

$$
R_{n}(\Delta)^{2} \leq \frac{K^{2} \Delta}{L^{*}} e^{3 L^{*}(n+1) \Delta}, \quad n=0,1, \ldots
$$

for $\Delta$ sufficently small, and thus

$$
R_{n}(\Delta)^{2} \leq \frac{K^{2} \Delta}{L^{*}} e^{3 L^{*}(T+\Delta)} \leq \frac{K^{2}}{L^{*}} e^{3 L^{*}(T+1)} \Delta, \quad n=0,1, \ldots, N_{T},
$$

for $\Delta$ sufficently small, since $N_{T} \Delta=T$ and we can assume without loss of generality that $\Delta \leq 1$.

This proves that the SIES has global discretization or convergence error of order at least one half.

\section{REFERENCES}

[1] L. Arnold, Random Dynamical Systems, Springer-Verlag (1997).

[2] T.A. Burton, Uniform asymptotic stability in functional differential equations, Proc. Amer. Math. Soc. 68(2) (1978), 195-199.

[3] T. Caraballo \& J.A. Langa, On the upper semicontinuity of cocycle attractors for nonautonomous and random dynamical systems, Dynamics of Continuous, Discrete and Impulsive Systems, Series A 10(2003), 491-513.

[4] T. Caraballo, J.A. Langa \& J.C. Robinson, Attractors of delay equations with variable delay, J. Math. Anal. Appl., 260 (2001), 421-438.

[5] T. Caraballo, P. Marín-Rubio \& J. Valero, Autonomous and non-autonomous attractors for differential equations with delays, J. Diff. Eqns. 208(2005), 9-41. 
[6] D. Cheban, P. E. Kloeden \& B. Schmalfuss, Pullback attractors in dissipative nonautonomous differential equations under discretization. J. Dyn. Systems \& Diff. Eqns. 13 (2001), 185-213.

[7] D. Cheban, P.E. Kloeden \& B. Schmalfuss, The relationship between pullback, forwards and global attractors of nonautonomous dynamical systems, Nonlinear Dynamics 83 Systems Theory 2(2002), 9-28.

[8] H. Crauel \& F. Flandoli, Attractors for random dynamical systems, Probability Theory and Related Fields 100(1994), 365-393.

[9] H. Crauel, A. Debussche \& F. Flandoli, Random attractors, J. Dyn. Diff. Eq. 9(1995), No. $2,307-341$.

[10] J.K. Hale, Asymptotic Behavior of Dissipative Systems, Math. Surveys and Monographs, AMS, Providence, (1988).

[11] J.K. Hale, Theory of Functional Differential Equations, Springer-Verlag, New York, (1977).

[12] J.K. Hale \& S.M. Verduyn-Lunel, Introduction to Functional Differential Equations, Springer-Verlag, New York, (1993).

[13] P. E. Kloeden, H. Keller \& B. Schmalfuss, Towards a theory of random numerical dynamics. In H. Crauel \& V. M. Gundlach (eds.), Stochastic Dynamics, Springer-Verlag, 1999. pp. 259-282.

[14] P.E. Kloeden \& D.J. Stonier, Cocycle attractors in nonautonomously perturbed differential equations, Dynamics Continuous, Discrete and Impulsive Systems 4(1998), no. 2, 211-226.

[15] B. Schmalfuss, Backward cocycle and attractors of stochastic differential equations, in V. Reitmann, T. Redrich \& N. J. Kosch (eds.), International Seminar on Applied MathematicsNonlinear Dynamics: Attractor Approximation and Global Behaviour (1992), 185-192.

[16] A.M. Stuart \& A.R. Humphries, Dynamical Systems and Numerical Analysis, Cambridge University Press, Cambridge, 1996.

E-mail address, Tomás Caraballo: caraball@us.es

E-mail address, Peter E. Kloeden: kloeden@math.uni-frankfurt.de

E-mail address, José Real: jreal@us.es

(Tomás Caraballo and José Real) Dpto. Ecuaciones Diferenciales y AnÁlisis Numérico, Universidad de Sevilla, Apdo. de Correos 1160, 41080-Sevilla (Spain)

(Peter E. Kloeden) Institut für Mathematik, Johann Wolfgang Goethe-Universität, D60054 Frankfurt am Main, Germany 